\title{
Precision Angular Measurements Using Scale of Chords
}

\author{
Dr A. M. Chandra \\ (Department of Civil Engineering, Sharda, University, India)
}

\begin{abstract}
Presently angular measurements are made using protractors having a normal accuracy of $1^{\circ}$ or at the most $1 / 2^{\circ}$. The scale of chords, as linear scale, can be constructed for measurement and construction of angles having accuracy equal to that of a normal protractor. This paper presents a new concept of construction of a diagonal scale of chords that can have an accuracy of 10' or less, and thus, using diagonal scale of chords, angles can be constructed or measured to a higher accuracy which is not possible using normal size protractors. Keywords: Angle measurements, Protractor, Scale of chords, Diagonal scale of chords
\end{abstract}

\section{Introduction}

For the measurement and construction of angles, protractor is the most convenient commonly used tool. The normal size protractors have their least count as $1^{\circ}$, but the bigger size protractors can have $1 / 2^{\circ}$ or $30^{\prime}$. To increase the least count further, the size of the protractor has to be increased making its use inconvenient.

Another method of measuring or constructing angles is using a scale of chords which is a linear scale. The plain scale of chords can have a least count which can be equal to that of a protractor. The least count of the plain scale of chords can be increased by making it a diagonal scale of chords without increasing its length. It is a new concept in measuring and constructing angles manually to higher accuracy which was not possible earlier.

\section{Plain Scale Of Chords}

To construct a plain scale of chords, a $90^{\circ}$ arc $A B^{\prime}$ (the arc subtending an angle of $90^{\circ}$. at its centre) as shown in Fig. 1, of convenient radius is divided into nine equal parts $A-1^{\prime}, 1^{\prime}-2^{\prime}, 2^{\prime}-3^{\prime}$, etc., each part subtending $10^{\circ}$ at the centre $O$ [Chandra and Chandra, 2003]. The division of an arc into odd number of equal divisions is done by the trial and error method using a bow divider. A new method of dividing an arc into odd number of equal divisions has been developed by the author and is going to be published soon.

The line $A O$ is extended to a convenient length $A C$. The points 1', 2', 3', etc., on the arc $A B^{\prime}$ are transferred to their corresponding points $1,2,3$, etc., on the line $A C$ by drawing arcs of radii $A-1^{\prime}, A-2^{\prime}, A-3^{\prime}$, etc., having centre at $A$. Each division $A-1,1-2,2-3$, etc., corresponds to an angle of $10^{\circ}$. By dividing each division into five equal parts, the scale of chords, so constructed, has a least count of $2^{\circ}$, and if it is possible to divide these divisions into 10 equal parts, the least count will be $1^{\circ}$. Similarly, if a bigger arc $A B^{\prime}$ is taken it may be possible to get a least count of $1 / 2^{\circ}$ or $30^{\prime}$, but practically it may not be possible to get a least count less than $30^{\prime}$ due to inconvenience in use of the scale as its size becomes too large, though theoretically it is possible.

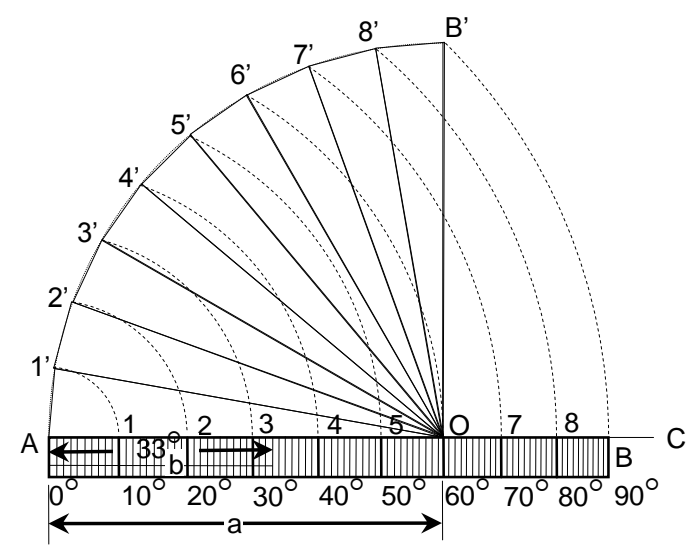

Fig. 1 Plain scale of chords

Now to construct an angle of say $33^{\circ}$ using the scale of chords, draw a line $P Q$ equal to $A O$, i.e., $a$, and then draw the $\operatorname{arc} Q M$ of radius $a$ with centre at $P$ (Fig. 2). On this arc, mark the point $K$ at a distance equal to $b$ from $Q$, the distance corresponding to $33^{\circ}$ as shown in Fig. 1. Join $P$ and $K$, and the angle $K P Q$ is equal to $33^{\circ}$. 


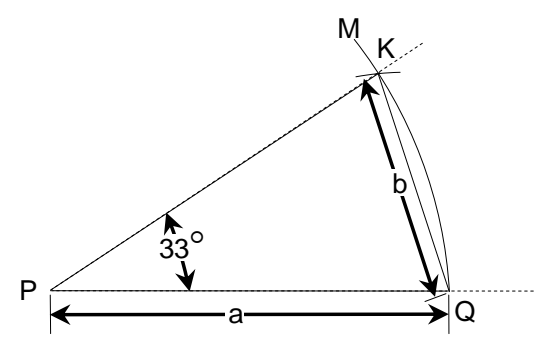

Fig. 2 Constructing an angle using scale of chords

If it is required to measure an angle $M P N(\theta)$ shown in Fig. 3, extend the line $P N$ to $Q$ such that $P Q$ is equal to $a$. Now draw an arc of radius $a$ with centre at $P$, intersecting the extension of $P M$ at $K$. Take a bow divider and measure the distance $Q K$ equal to $c$. Lay down this measured distance $c$ on the scale of chords which will give the value of the angle as $41^{\circ}$ in this case.

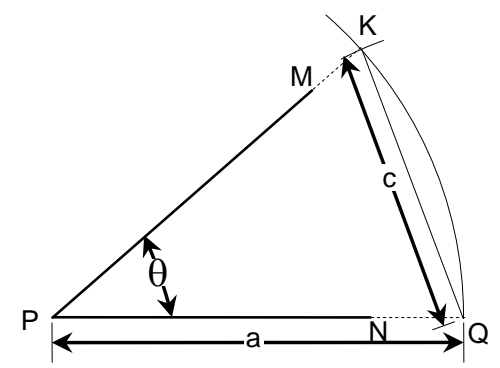

Fig. 3 Measuring an angle using scale of chords

\section{Diagonal Scale Of Chords}

Fig. 4 shows a diagonal scale of chords $A P Q B$. The base $A B$ is a part of the plain scale of chords, and its construction is explained above. Now to convert this plain scale of chords into a diagonal scale of chords, draw a perpendicular $A P$ to $A B$ of a convenient length at $A$, and complete the rectangle $A P Q B$. Divide $A P$ into six equal divisions, and draw lines for each division parallel to $A B$. Also extend the lines of each division of $1^{\circ}$ of the plain scale of chords up to the line $P Q$, and draw the diagonals for each $1^{\circ}$ division as shown in the figure. This construction now provides the least count of $10^{\prime}$. The horizontal distances $b$ and $c$ more precisely indicate the angles as $33^{\circ} 40^{\prime}$ and $41^{\circ} 20^{\prime}$, respectively.

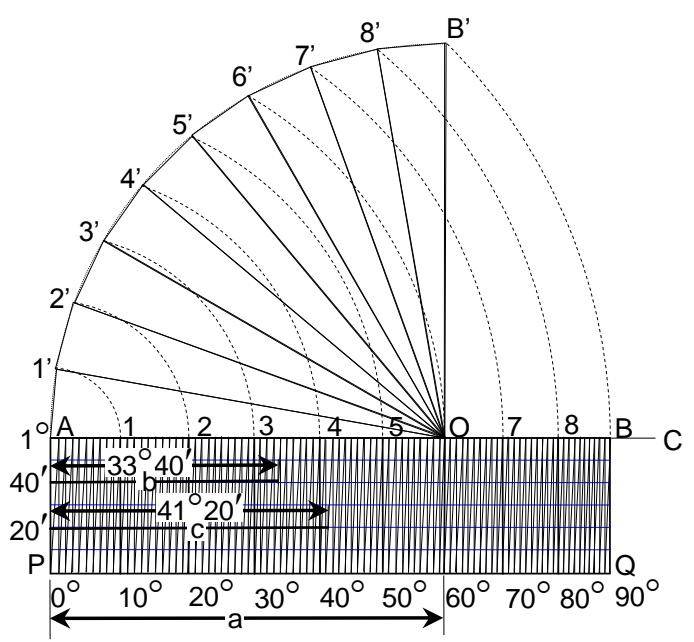

Fig. 4 Diagonal scale of chords

Construction and measurement of angles to an accuracy of $10^{\prime}$ can be done using the diagonal scale of chords in a similar manner as in the case of plain scale of chords explained above. 


\section{Practical Considerations}

There are two important points which require attention. The first point to be noted is that the divisions $A-1,1-2,2-3$, etc., are not equal in length. Though the chord lengths $A-1^{\prime}, 1^{\prime}-2^{\prime}, 2^{\prime}-3^{\prime}$, etc., are equal, the distance $A-2$ is not equal to $\left[\left(A-1^{\prime}\right)+\left(1^{\prime}-2^{\prime}\right)\right]$, and so on. With the same logic, the subdivisions of the divisions $A-1,1-2,2-3$, etc., are also not equal. Since the difference between the lengths of two subdivisions is so small that it cannot be measured, they may be treated to be equal from practical consideration of plotting accuracy.

Another point to be noted that the line $A P$ shown in Fig. 4, can be divided into 12 equal parts giving the least count of the scale as $5^{\prime}$, but the difference between the linear distance for $5^{\prime}$ and $10^{\prime}$ will be so small that it cannot be measured, and therefore, angles having difference of $5^{\prime}$ cannot be measured. Thus dividing the line $A P$ into 12 equal parts is not going to improve the accuracy of the scale until $A P$ and $A B$ are taken sufficiently large, which will make the scale inconvenient to use.

\section{Conclusions}

A new concept of diagonal scale of chords presented here has its own practical use for construction and measurement of angles manually to an accuracy which is not achievable using a normal protractor. The diagonal scale of chords can be manufactured like other scales and can be made available in market to achieve higher accuracy in angular measurements.

\section{Acknowledgements}

The author is grateful to the Arba Minch University, Ethiopia, where he was working teaching assignment, for all help and conducive environment provided by the university to write this technical paper during 2002-04.

\section{References}

[1] A. M. Chandra and Satish Chandra, Engineering Graphics (Narosa Publishing House, New Delhi, 2003) 